\title{
Thrombotic Microangiopathy After Allogeneic Hematopoietic Stem Cell Transplantation: An Autopsy Study
}

\author{
Koushan Siami, ${ }^{1}$ Kiarash Kojouri, ${ }^{2}$ Karen K. Swisher, ${ }^{3}$ George B. Selby, ${ }^{2}$ James N. George, ${ }^{2}$ \\ and Zoltan G. Laszik ${ }^{1,4}$
}

\begin{abstract}
Background. Posttransplantation thrombotic microangiopathy (PTMA) is a complication of allogeneic hematopoietic stem cell transplantation (HSCT). However, limited autopsy data are available, and it remains unclear whether PTMA is a discrete clinical and pathologic entity. The aims of this autopsy study were to determine the correlation between clinical and pathologic diagnosis of PTMA, to define the precise morphologic spectrum of PTMA, and to seek for potential etiologic factors.

Methods. The study included 20 consecutive patients with HSCT autopsied at the University of Oklahoma, between 1994 and 2005. Applying strict clinical-laboratory criteria, 6 patients were diagnosed clinically with PTMA and treated with plasma exchange. Clinical variables, including underlying disease, conditioning regimen, stem cell donor status, duration and serum level of cyclosporine, infections, and acute graft versus host disease were compared statistically in patients with histologic signs of PTMA $(n=8)$ with those without PTMA $(n=12)$.

Results. PTMA was verified histologically in all 6 patients with a clinical diagnosis of PTMA but only 2 of the 14 patients who were not clinically diagnosed had histologic evidence of PTMA $(P<0.0001)$. Kidneys were affected in all 8 patients with PTMA, and limited extrarenal involvement by PTMA was observed in 3 of these 8 patients. No statistically significant differences in relevant clinical and morphologic variables were identified between the PTMA and nonPTMA groups.

Conclusions. This study documents a strong correlation between the clinical and morphologic diagnosis of PTMA. The kidney is the primary target of PTMA, with dominant glomerular and arteriolar involvement. The etiology is likely to be multifactorial.
\end{abstract}

Keywords: Posttransplantation thrombotic microangiopathy, Allogeneic hematopoietic stem cell transplantation.

(Transplantation 2008;85: 22-28)

$\mathrm{T}$ hrombotic microangiopathy (TMA) has been considered to be a complication of allogeneic hematopoietic stem cell transplantation (HSCT) (1-3), which is described as posttransplantation TMA (4). However, there is little evidence to establish posttransplantation TMA as a specific clinical or pathologic entity (5). After allogeneic HSCT, patients may have many critical complications, making the clinical diagnosis of TMA uncertain (5). This uncertainty is emphasized by the extreme variability of the reported frequency of the diagnosis of posttransplantation TMA after allogeneic HSCT, ranging from $0.5 \%$ to $63.6 \%$, and the use of many different sets of diagnostic criteria (5). This striking variation may be caused by the difficult clinical diagnosis of TMA in these patients. Microangiopathic hemolytic anemia and thrombocytopenia, the principal clinical and laboratory diagnostic features of TMA, can be mimicked by common complications in patients

\footnotetext{
${ }^{1}$ Department of Pathology, University of Oklahoma Health Sciences Center, 940 Stanton L. Young Blvd., Oklahoma City, OK 73190.

${ }^{2}$ Section of Hematology/Oncology, University of Oklahoma Health Sciences Center, Oklahoma City, OK.

${ }^{3}$ Department of Medicine, University of Oklahoma Health Sciences Center, Oklahoma City, OK.

${ }^{4}$ Address correspondence to Zoltan G. Laszik, Department of Pathology, University of California San Francisco, 513 Parnassus Ave., Room S566, San Francisco, CA 94143.

E-mail: zoltan.laszik@ucsfmedctr.org

Received 30 April 2007. Revision requested 28 June 2007.

Accepted 19 September 2007.

Copyright $\odot 2008$ by Lippincott Williams \& Wilkins

ISSN 0041-1337/08/8501-22

DOI: 10.1097/01.tp.0000297998.33418.7e
}

after HSCT, such as opportunistic infections, chemotherapy regimen-related toxicity, radiation-related injury, and acute graft-versus host-disease (GVHD).

In addition to the uncertain clinical data on posttransplantation TMA, detailed morphologic descriptions of autopsy findings in patients with the clinical diagnosis of posttransplantation TMA are sparse, and the extent and morphologic severity of TMA have not been well-characterized. A recent systematic review identified reports of autopsies in only 35 patients who had been diagnosed with TMA after allogeneic HSCT; none had systemic TMA; when TMA was described, it was localized to the kidneys; infection was the most commonly reported cause of death (5).

To determine whether the pathologic features of TMA are related to the clinical diagnosis of posttransplantation TMA, we analyzed the clinical and pathologic data of 20 consecutive patients who had autopsies after allogeneic HSCT. Six of the 20 patients had been clinically diagnosed with posttransplantation TMA and treated with plasma exchange. The aims of the study were (1) to correlate the clinical diagnosis of posttransplantation TMA with the autopsy findings, (2) to correlate clinical parameters with autopsy findings of TMA to identify potential etiologic factors for the pathogenesis of posttransplantation TMA, (3) to compare the autopsy findings of posttransplantation TMA with the classic morphologic findings of thrombotic thrombocytopenic purpura (TTP) (i.e., multiple organ involvement by systemic TMA) and hemolytic uremic syndrome (HUS; i.e., dominant renal TMA with limited involvement of other organs), and (4) to 
analyze the renal morphologic findings of TMA in patients with posttransplantation TMA to determine which (if any) are the dominant morphologic patterns (i.e., glomerular versus arterial).

\section{MATERIALS AND METHODS}

\section{Patients}

All patients who died and underwent autopsy after receiving allogeneic HSCT at the Department of Pathology, University of Oklahoma Health Sciences Center between April 25, 1994, the date of the first autopsy examination of a patient after allogeneic HSCT, and December 31, 2005 were identified for this analysis.

\section{Clinical Data}

Age, gender, underlying disease and its status at the time of HSCT, conditioning regimen, use of total body irradiation as part of conditioning regimen, stem cell donor (related vs. unrelated), level of HLA antigen mismatch, duration of cyclosporine exposure, peak serum cyclosporine level, systemic cytomegalovirus (CMV) infection, systemic fungal infection, and acute GVHD, its maximum overall grade, and presence of active GVHD at the time of death were recorded for each patient.

Systemic CMV infection/reactivation was diagnosed clinically and required positive CMV antigenemia or viral culture. Systemic fungal infection was diagnosed clinically and required positive culture, staining, or histologic evidence of invasive fungal infection.

Acute GVHD was diagnosed based on clinical criteria (involvement of skin, gastrointestinal tract, and liver) and, if available, supported by histology. Active GVHD at the time of death was also separately recorded. GVHD was considered active if symptoms/signs attributable to GVHD were present and the patient received immunosuppressive therapy for treatment of GVHD within 30 days before death. Patients were classified as TMA and non-TMA groups using independent clinical and histologic criteria.

\section{Histologic Data}

Hematoxylin and eosin-stained sections of paraffinembedded autopsy tissues were reviewed by two pathologists (K.S. and Z.G.L.) independently for histologic evidence of thrombotic microangiopathy (TMA) in a blinded fashion, without clinical information regarding the presence or absence of a clinical diagnosis of posttransplantation TMA. Sections from heart, lungs, liver, pancreas, kidneys, adrenals, thyroid gland, spleen, esophagus, stomach, intestines, bladder, bone marrow, and brain were available for histologic evaluation from all 20 cases. Prostate and testes were also examined in males and ovaries and uterine tissues in females. Sampling of lungs included sections from each lobe, at least one section from both kidneys, and multiple sections from the brain; the rest of the organs were sampled representatively. From each kidney, periodic acid Schiff-stained sections were also prepared and examined. Histologic diagnosis of TMA in the kidney required the presence of thrombotic lesions and/or any other classic glomerular or vascular features of TMA, as listed in Table 1. Detailed morphologic assessment of the kidneys included examination of at least 200 glo-
TABLE 1. Histologic features of TMA in the kidney

Glomerular changes

Capillaries and capillary walls

Thickening of the wall and double contours of basement membranes

Narrowing and closure of capillary lumina

Fragmenting of red blood cells

Fibrin thrombi/fibrinoid necrosis

Mesangium

Edema and fibrillary appearance

Deposition of fibrin and fragmented red blood cells

Mesangiolysis

Vascular changes

Intimal swelling

Fibrinoid necrosis and/or thrombosis

Fragmented red blood cells in the wall

Myointimal cellular proliferation

meruli, 20 arterioles, and 20 interlobular arteries in each case. The diagnosis of TMA in other organs was based on thrombotic lesions in the capillaries and/or larger vessels.

Fungal infections suspected on hematoxylin and eosin-stained slides were confirmed by Grocott methenamine silver special stain. Among patients with systemic fungal infection, the subset of patients who had evidence of angioinvasive fungal infection (AFI) was further determined. AFI was defined as presence of fungal hyphae in the lumen of capillaries or small vessels, and/or traversing blood vessel walls.

\section{Clinical Classification}

Patients who had received plasma exchange for treatment of clinically diagnosed posttransplantation TMA (6) were assigned to the Clinical TMA group; the remainder of the patients comprised the Clinical non-TMA group. Necessary criteria for the clinical diagnosis of posttransplantation TMA were thrombocytopenia and microangiopathic hemolytic anemia without an apparent alternative etiology $(3,7,8)$. The criterion of plasma exchange treatment as the basis for assignment of patients to Clinical TMA and Clinical nonTMA groups was established because it indicated a firm commitment by the physician to the diagnosis of posttransplantation TMA $(7,8)$.

\section{Histologic Classification}

On the basis of the presence or absence of histologic evidence of TMA in the kidneys and other organs patients were also assigned to histologic TMA and histologic nonTMA groups. This assignment was independent of and without knowledge of the patient's clinical classification. For the histologic TMA group, percentages of renal glomeruli, arterioles and interlobular arteries showing features of TMA were calculated to assess the extent of involvement. The number of organs showing histologic features of TMA also was recorded.

\section{Statistical Analysis}

Association between clinical and histologic classifications was analyzed with Fisher's exact test. Histologic TMA 
and histologic non-TMA groups were compared for categorical and continuous variables, with chi-square (or Fisher's exact) and nonparametric Wilcoxon rank tests, respectively. Correlation between renal glomerular and arteriolar involvement was performed by using Pearson correlation co-efficient. SAS ${ }^{\circledR}$ software, version 8.0 (Cary, NC) was used for statistical comparisons.

\section{RESULTS}

Twenty-three consecutive patients were identified who had an autopsy after allogeneic HSCT. Three patients were excluded from this analysis, two because the autopsy was limited to examination of the brain and one because of missing slides and tissue blocks. The remaining 20 patients, 8 women and 12 men, with a median age of 36 years (range, 6-62 years) at the time of HSCT were included in this analysis. The diagnoses for which HSCT had been performed were acute lymphoblastic leukemia (five patients), acute myelogenous leukemia (three patients), chronic myelogenous leukemia (four patients), myelodysplasia (five patients), and multiple myeloma, non-Hodgkin lymphoma, and aplastic anemia (one patient each).

Six $(30 \%)$ of the 20 patients had plasma exchange treatment for clinically diagnosed posttransplantation TMA; 14 (70\%) did not. Review of autopsy slides demonstrated histologic evidence of TMA in 8 ( $40 \%$ ) of the 20 patients; whereas in $12(60 \%)$ others, there was no histologic evidence of TMA. A significant association existed between clinical diagnosis of posttransplantation TMA and histologic evidence of TMA. All 6 patients (100\%) with clinical diagnosis of TMA had histologic evidence of TMA, whereas only 2 of the 14 patients without clinical features of TMA (14\%) had histologic evidence of TMA (2-tailed $P<0.0001$; Table 2). The diagnosis of posttransplantation TMA was not considered in those two patients because the overt clinical complications of the HSCT had preempted consideration of the diagnosis of postransplantation TMA and of treatment with plasma exchange. One patient was a 36-year-old man with chronic phase chronic myelogenous leukemia who had received a sibling donor HSCT. His terminal complications included bacterial and CMV sepsis and grade 4 GVHD with bowel necrosis. He died 4 months after HSCT. The other patient was a 39-year-old woman with myelodysplasia who had received an unrelated donor HSCT. She also had bacterial sepsis and grade 4 GVHD as her terminal complications and died 2 months after HSCT, without ever leaving the hospital.

\section{Patients With Histologic TMA}

Among the eight patients with histologic evidence of TMA, the kidneys were involved in all patients, with median

TABLE 2. Association between clinical diagnosis of posttransplantation TMA and histologic findings of TMA (two-tailed $P<0.0001$, Fisher exact test)

\begin{tabular}{lcc} 
& $\begin{array}{c}\text { Clinical TMA } \\
(\mathbf{n}=\mathbf{6 , 3 0 \% )}\end{array}$ & $\begin{array}{c}\text { Clinical non-TMA } \\
(\mathbf{n}=\mathbf{1 4}, \mathbf{7 0})\end{array}$ \\
\hline $\begin{array}{c}\text { Histologic TMA } \\
(\mathrm{n}=8,40 \%)\end{array}$ & 6 & 2 \\
$\begin{array}{c}\text { Histologic non-TMA } \\
(\mathrm{n}=12,60 \%)\end{array}$ & 0 & 12 \\
\hline
\end{tabular}

$74 \%$ (range, $34-85 \%$ ) of glomeruli and median $78 \%$ (range, $33-91 \%$ ) of arterioles showing diagnostic pathologic features of TMA (Fig. 1; Table 3). There was also significant correlation between the extent of glomerular and arteriolar involvement $(P=0.0254$; Fig. 2$)$. The extent of glomerular and arteriolar changes was similar between the six patients who had been clinically diagnosed with posttransplantation TMA and the two patients who had histologic evidence of TMA but who had not been clinically diagnosed with posttransplantation TMA. In all eight patients, vascular changes of TMA were less frequent in the renal interlobular arteries than in the arterioles. There was no correlation between the extent of arterial TMA on one hand and glomerular or arteriolar TMA on the other hand.

Extrarenal organ involvement was observed in only three patients. All three were among the eight patients who also had renal involvement with TMA, and all three had also been clinically diagnosed with TMA. One patient had limited involvement of the pancreas; one patient had limited involve-

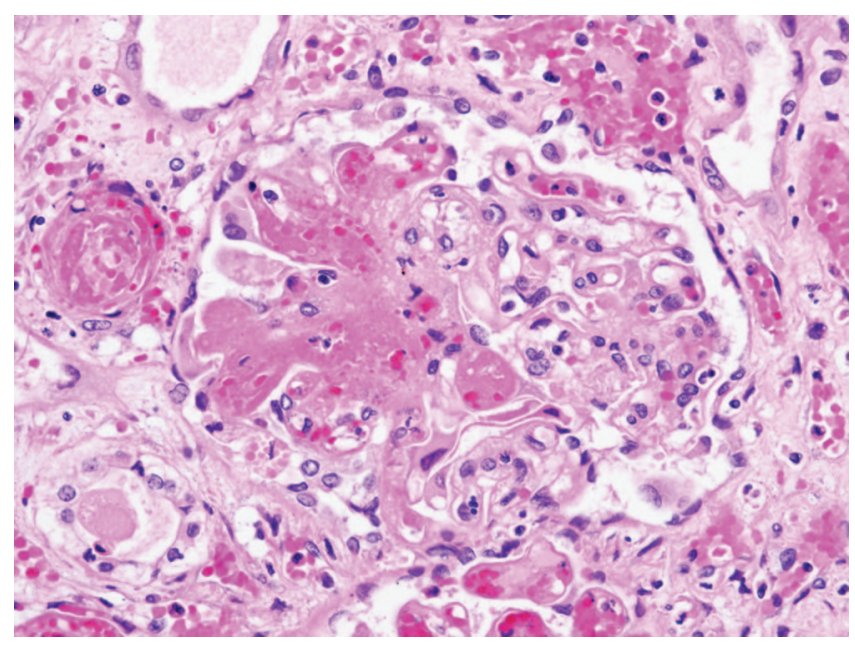

FIGURE 1. Thrombotic microangiopathy with glomerular capillary thrombi and fibrin and fragmented red blood cells in the mesangial area. The arteriole on the left shows thrombotic occlusion.

TABLE 3. Extent of glomerular, arteriolar and interlobular arterial involvement in kidney sections of patients with Histologic TMA $(n=8)$

\begin{tabular}{lccc} 
Case & $\begin{array}{c}\text { Glomerular } \\
\text { TMA, \% }\end{array}$ & $\begin{array}{c}\text { Arteriolar } \\
\text { TMA, \% }\end{array}$ & $\begin{array}{c}\text { Arterial } \\
\text { TMA, \% }\end{array}$ \\
\hline 1 & 84 & 91 & 4 \\
2 & 34 & 33 & 0 \\
3 & 75 & 87 & 6 \\
4 & 85 & 75 & 6 \\
5 & 67 & 69 & 8 \\
6 & 85 & 80 & 4 \\
$7^{a}$ & 55 & 84 & 10 \\
$8^{a}$ & 73 & 71 & 9 \\
\hline
\end{tabular}

Percentages indicate percent involvement of the counted glomeruli $(\geq 200)$, arterioles $(\geq 20)$, and interlobular arteries $(\geq 20)$ by TMA.

${ }^{a}$ Clinical non-TMA patient. 


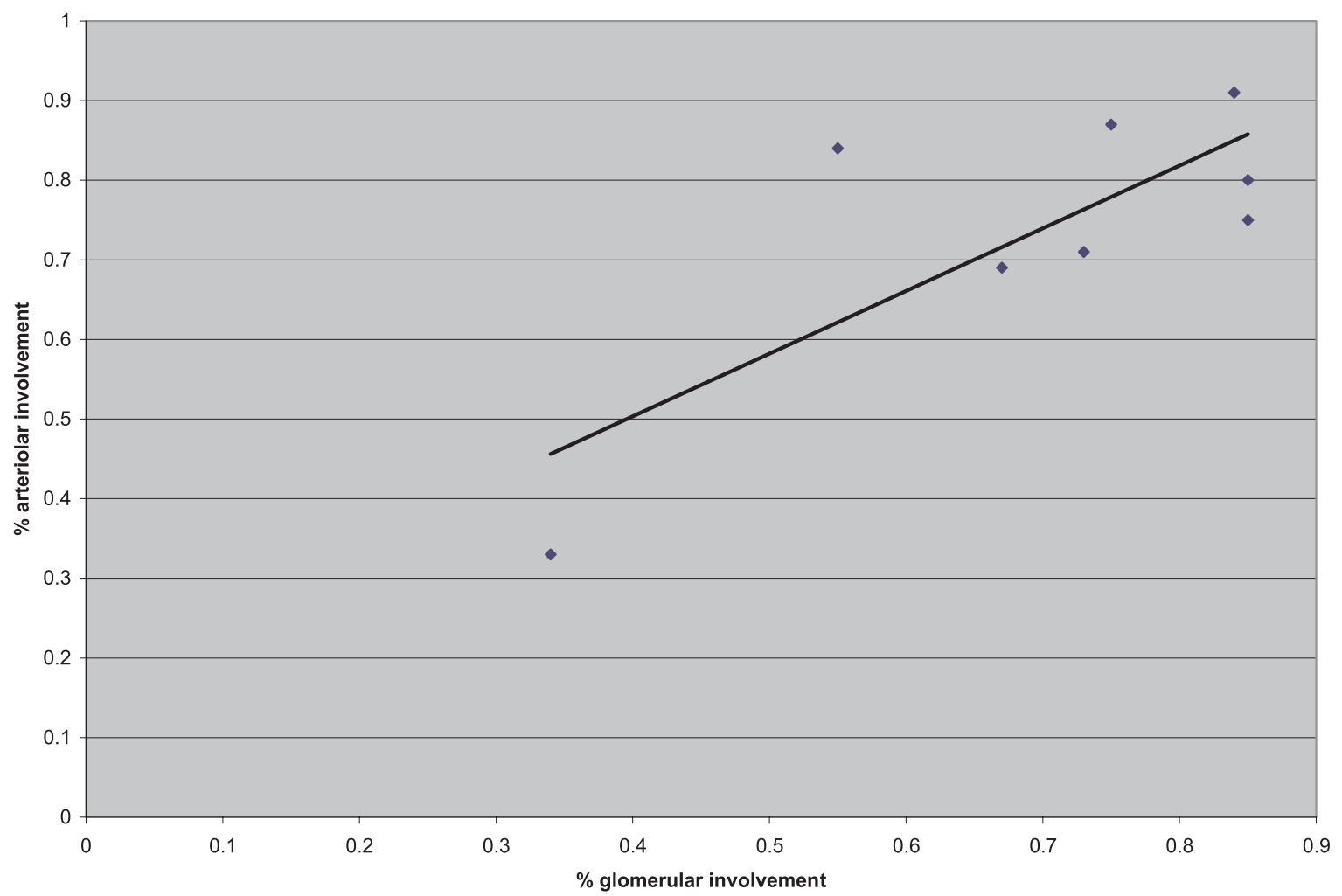

FIGURE 2. Correlation between glomerular TMA and arteriolar TMA in patients with Histologic TMA ( $\mathrm{n}=8$, Pearson's rank correlation coefficient $0.77, P=0.0254)$.

ment of the lung; and one patient had limited involvement of both the lung and heart. No features of TMA were observed in any other organs including the brain and the intestines.

Patients with histologic evidence of TMA (histologic TMA group) were compared with those without TMA (histologic non-TMA group) for variables that might be associated with the occurrence of TMA (Table 4). Six (75\%) of the eight patients in the histologic TMA group versus only 4 of 12 patients $(33 \%)$ in the histologic non-TMA group had unrelated donors. Four (50\%) patients in the histologic TMA group had one antigen mismatch, whereas all patients in the histologic non-TMA group, except for one patient who received cord blood stem cell transplant, had a full antigen match transplant. All eight patients (100\%) in the histologic TMA group were diagnosed with acute GVHD, and six (75\%) of them had evidence of active GVHD at the time of their death. In the histologic non-TMA group, 8 of 12 patients (67\%) developed GVHD and $4(33 \%)$ had active GVHD at the time of death. Systemic CMV infection/reactivation and AFI each occurred among five of eight histologic TMA patients $(63 \%)$, whereas only 3 and 4 of 12 histologic non-TMA patients (25\% and $33 \%)$ had developed CMV infection/reactivation and AFI, respectively. Five $(63 \%)$ patients in the histologic TMA group had received total body irradiation as part of their preparative regimen; three had not. Five (63\%) patients in the histologic TMA group and four (33\%) patients in the histologic non-TMA group had angioinvasive fungal infection. Although these observations suggested that patients with histologic TMA had a greater rate of risk factors and complications, none of the differences were statistically significant.

\section{Patients With Clinical TMA}

In all 6 patients in the clinical TMA group, the diagnosis of posttransplantation TMA was made from 20 days to 7 months (median, 2.5 months) after HSCT, and these patients died from 2 to 45 days (median, 18 days) after the first plasma exchange treatment for the diagnosis of posttransplantation TMA. ADAMTS13 activity was determined at the time of the clinical diagnosis of TMA for the three most recent patients; their levels were $80 \%, 100 \%$, and 75\%, respectively.

\section{Cause of Death}

Systemic infections were the cause of death in 15 of the 20 patients. The etiologies of infection were aspergillus (seven patients), candida (four patients), CMV (two patients), and bacterial sepsis (two patients). Three patients died of pulmonary complications; principal causes of death were listed as interstitial pneumonia, diffuse alveolar damage and hemorrhage, and adult respiratory distress syndrome, each for one patient. In one patient the principal cause of death was acute GVHD and one died of brain parenchymal hemorrhage. All eight patients with histologic TMA, including the six patients with clinical TMA, died of infection. In none of the eight patients did TMA appear to contribute significantly to the death of the patient. Additional significant autopsy findings included pathologic changes in the gastrointestinal tract and liver (GVHD, seven patients; venoocclusive disease, two pa- 
TABLE 4. Comparison of certain clinical and histologic variables between histologic TMA and histologic non-TMA groups

\begin{tabular}{|c|c|c|c|}
\hline & $\begin{array}{l}\text { Histologic TMA } \\
\qquad(\mathrm{n}=8)\end{array}$ & $\begin{array}{l}\text { Histologic non-TMA } \\
\qquad(\mathrm{n}=12)\end{array}$ & One-tailed $P$ value $^{a}$ \\
\hline Age, years, median (range) & $34(17-54)$ & $37(6-62)$ & NS \\
\hline Gender, F;M & $3 ; 5$ & $5 ; 7$ & NS \\
\hline \multicolumn{4}{|l|}{ Disease status at HSCT } \\
\hline In remission & 2 & 6 & NS \\
\hline Not in remission & 6 & 5 & \\
\hline Not applicable (aplastic anemia) & 0 & 1 & \\
\hline \multicolumn{4}{|l|}{ Conditioning regimen ${ }^{b}$} \\
\hline Busulfan/cyclophsphamide & 3 & 5 & NS \\
\hline Cyclophosphamide/TBI & 5 & 6 & \\
\hline Melphalan/fludarabine & & 1 & \\
\hline Donor & & & NS \\
\hline Related & 2 & 8 & $(0.0849)$ \\
\hline Unrelated & 6 & 4 & \\
\hline Level of mismatch & & & NS \\
\hline Full match & 4 & 11 & $(0.0578)$ \\
\hline $1 / 6$ mismatch & 4 & 0 & \\
\hline $2 / 6$ mismatch $^{c}$ & 0 & 1 & \\
\hline \multicolumn{4}{|l|}{ CsA } \\
\hline No. patients & 8 & 11 & NS \\
\hline Peak level, median (range) & $822(239-1297)$ & $819(346-1716)$ & NS \\
\hline Duration, ays; median, (range) & $48(16-222)$ & $72(8-130)$ & NS \\
\hline GVHD & 8 & 8 & NS \\
\hline Active GVHD at the time of death & 6 & 4 & 0.0849 \\
\hline CMV infection & 5 & 3 & NS \\
\hline Systemic fungal infection & 5 & 6 & NS \\
\hline AFI & 5 & 4 & NS \\
\hline
\end{tabular}

${ }^{a}$ One-tailed $\mathrm{p}$ values $<0.1$ are mentioned (with assumption that TMA patients are more likely to have unrelated donor, tissue antigen mismatch, and GVHD).

${ }^{b}$ Patients in the TMA group also received etoposide as part of their conditioning regimen, one patient in the non-TMA group also received anti-thymocyte globulin as part of her conditioning regimen.

${ }^{c}$ The patient with 2/6 mismatch (one B and one Dr allele) received umbilical cord blood stem cell transplantation.

tients; drug hepatotoxicity, one patient; prominent cholestasis, five patients; and portal fibrosis, three patients), systemic posttransplantation lymphoproliferative disorder (one patient), and recurrence of acute myelogenous leukemia with multiorgan involvement (one patient).

\section{DISCUSSION}

In this clinical and pathologic study of posttransplantation TMA, a detailed morphologic autopsy analysis was performed in twenty consecutive patients who died after allogeneic HSCT. Morphologic analyses were performed without knowledge of the clinical diagnosis of TMA, which had been made in 6 of the 20 patients. All six patients clinically diagnosed with TMA had thrombocytopenia and microangiopathic hemolytic anemia that could not be attributed to an alternative transplant-related complication and all had been treated with plasma exchange, emphasizing commitment to the diagnosis of posttransplantation TMA. This blinded clinical and pathologic approach allowed us to correlate the clinical diagnosis with the morphologic findings and to morphologically validate the clinical diagnosis.
The results of our study demonstrated a surprisingly strong correlation between the clinical and autopsy morphologic findings. Our a priori hypothesis was that a correlation between clinical and autopsy morphologic findings would be weak or nonexistent, because of the difficulty and inconsistency of the clinical diagnosis (5) as well as because of the multiple potential etiologies for histologic evidence of TMA. For example, we have previously documented that the clinical diagnosis of posttransplantation TMA was significantly correlated with who the patient's physician was, suggesting a high degree of subjectivity of the diagnosis (6). However, in the current study, all six patients who were diagnosed clinically with posttransplantation TMA also had morphologic features of TMA. Autopsy also documented morphologic features of TMA in two patients who were not clinically diagnosed with TMA. In these two patients review of the clinical data demonstrated critical complications of sepsis and GVHD in addition to the histologic features of TMA, which were limited to the kidneys.

Our study assessing the autopsy findings in a series of 20 consecutive autopsies in patients after allogeneic HSCT 
identified histologic evidence of TMA in 8 patients (40\%), all of whom showed features of TMA in the kidneys. The two prototypical forms of TMA are hemolytic-uremic syndrome (HUS) and thrombotic thrombocytopenic purpura (TTP). Renal involvement is usually more severe in HUS while multi-organ involvement with extensive capillary microthrombi is the typical finding for TTP $(9,10)$. Although subtle histologic differences do exist between HUS and TTP, $(9,11)$ histologic assessment is generally considered to be unreliable to separate the two entities. Our findings on patients after allogeneic HSCT documented dominant renal morphologic manifestations of TMA, a pattern more reminiscent of HUS rather than TTP. Limited lung, pancreas, and heart involvement were observed only in three of our eight cases. Therefore, our findings confirm the previous observations that the kidney is the primary target of TMA after allogeneic HSCT (5).

In previous pathologic studies on patients with TMA unrelated to HSCT, the various morphologic patterns of TMA in the kidney (predominantly glomerular involvement, predominantly arterial involvement, and mixed forms) have been shown to correlate well with the clinical presentation and outcome $(12,13)$. In general, the outcome is better in patients who have predominantly glomerular involvement and worse in those with predominantly vascular involvement. Our cases of posttransplantation TMA demonstrated predominantly glomerular and arteriolar involvement and little or no involvement of interlobular size arteries. Applying these previous observations on the correlation between clinical and pathologic findings to patients with posttransplantation TMA, $(12,13)$ a relatively good renal prognosis may be expected. Consistent with this interpretation, none of the patients in this series who had morphologic evidence of renal TMA died of renal failure. The findings of dominant glomerular and arteriolar involvement are also consistent with previously published descriptions of posttransplantation TMA (14-16).

The etiology of posttransplantation TMA is unknown. Because of the close resemblance of the renal morphologic findings in posttransplantation TMA to radiation nephropathy, radiation has long been suspected to be the most significant factor in the pathogenesis $(15,16)$. The deleterious effects of radiation might be potentiated by the cytotoxic effects of chemotherapy conditioning regimens (15). An important observation of our study is identification of three patients with histologic evidence of posttransplantation TMA but without previous radiation therapy. This finding clearly indicates that posttransplantation TMA can develop without radiation, and therefore, should not be considered as a form of radiation nephropathy. However, a number of additional factors, such as cyclosporine and tacrolimus treatment, cytomegalovirus, fungal and Helicobacter pylori infections, acute GVHD, and HLA-mismatched transplants have also been considered in the pathogenesis $(9,10)$. Our findings did not demonstrate a statistically significant correlation between posttransplantation TMA and any of the clinical-laboratory parameters examined: age, gender, total body radiation, conditioning regimen, donor status (related vs. unrelated), HLA mismatch, CMV infection, systemic fungal infections, angioinvasive fungal infections, GVHD, and active GVHD at the time of death. The only clinical variable that approached statistical significance was the donor status (one-tailed
$P=0.0578)$. Although this cohort is currently the largest published experience, the number of patients with TMA is small. A larger experience may provide more power to document potential etiologic correlations.

ADAMTS13 activity was measured in only three of our six patients with a clinical diagnosis of TMA. Although severe ADAMTS13 deficiency is a well-defined etiology of TTP, (3) the absence of ADAMTS13 deficiency in our three patients is consistent with the previous reports on patients with posttransplantation TMA (17-19). The absence of ADAMTS13 deficiency in patients with posttranplantation TMA has been considered as one of the potential causes of ineffectiveness of plasma exchange treatment in these patients (2). However, Vesely et al. (7) showed that severe ADAMTS13 deficiency does not identify all patients diagnosed with TMA (HUS/TTP) who may respond to plasma exchange treatment.

Our data are the first to clearly document the correlation of clinical and pathologic abnormalities of posttransplantation TMA. Although the clinical diagnosis is often uncertain, all 6 of the patients who had been clinically diagnosed with posttransplantation TMA had histologic evidence of TMA at autopsy. The histologic abnormalities of TMA were almost exclusively limited to the kidney. This distinguishes posttransplantation TMA from TTP and supports the clinical impression that plasma exchange treatment may not be effective for these patients $(2,5)$. Although the number of patients in our cohort is small, our data support the concept that posttransplantation TMA is a discrete clinical and pathologic entity, distinct from other commonly recognized transplantrelated complications.

\section{REFERENCES}

1. Ruutu T, Barosi G, Benjamin RJ, et al. Diagnostic criteria for hematopoietic stem cell transplantation-associated microangiopathy (TAM): Results of a consensus process by an International Working Group. Haematologica/The Hematol J 2007; 92: 95.

2. Kojouri K, George JN. Thrombotic microangiopathy following allogeneic hematopoietic stem cell transplantation. Curr Op Oncol 2007; 19: 148.

3. George JN. Thrombotic thrombocytopenic purpura. NEng J Med 2006; 354: 1927.

4. Ho VT, Cutler C, Carter S, et al. Blood and marrow transplant clinical trials network toxicity committee consensus summary: Thrombotic microangiopathy after hematopoietic stem cell transplantation. Biol Blood Marrow Transplant 2005; 11: 571.

5. George J, Li X, McMinn JR, et al. Thrombotic thrombocytopenic purpura-hemolytic uremic syndrome following allogeneic HPC transplantation: A diagnostic dilemma. Transfusion 2004; 44: 294.

6. Roy V, Rizvi MA, Vesely SK, George JN. Thrombotic thrombocytopenic purpura-like syndromes following bone marrow transplantation: An analysis of associated conditions and clinical outcomes. Bone Marrow Transplantation 2001; 27: 641 .

7. Vesely SK, George JN, Lammle B, et al. ADAMTS13 activity in thrombotic thrombocytopenic purpura-hemolytic uremic syndrome: Relation to presenting features and clinical outcomes in a prospective cohort of 142 patients. Blood 2003; 101: 60.

8. George JN, Vesely SK, Terrell DR. The Oklahoma thrombotic thrombocytopenic purpura-hemolytic uremic syndrome (TTP-HUS) registry: A community perspective of patients with clinically diagnosed TTP-HUS. Semin Hematol 2004; 41: 60.

9. Laszik Z, Silva FG. Hemolytic-uremic syndrome, thrombotic thrombocytopenic purpura and systemic sclerosis. In: Jennette JC, Olson JL, Schwartz MM, Silva FG, eds. Heptinstall's Pathology of the Kidney (6th ed). Philadelphia: Lippincott Williams \& Wilkins; 2007: 701. 
10. Sarkodee-Adoo C, Sotirescu D, Sensenbrenner L, et al. Thrombotic microangiopathy in blood and marrow transplant patients receiving tacrolimus and cyclosporine A. Transfusion 2003; 43: 78.

11. Hosler GA, Cusumano AM, Hutchins GM. Thrombotic thrombocytopenic purpura and hemolytic uremic syndrome are distinct pathologic entities. A review of 56 autopsy cases. Arch Pathol Lab Med 2003; 127: 834 .

12. Thoenes W, John HD. Endotheliotropic (hemolytic) nephroangiopathy and its various manifestation forms (thrombotic microangiopathy, primary malignant nephrosclerosis, hemolytic-uremic syndrome). Klin Wochenschr 1980; 58: 173.

13. Habib R, Lévy M, Gagnadoux M-F, Broyer M. Prognosis of the hemolytic uremic syndrome in children. Adv Nephrol 1982; 11: 99.

14. Iskandar SS, Browning MC, Lorentz WB. Mesangiolytic glomerulopathy in a bone marrow allograft recipient. Hum Pathol 1989; 20: 290 .
15. Antignac C, Gubler MC, Leverger G, Broyer M, et al. Delayed renal failure with extensive mesangiolysis following bone marrow transplantation. Kidney Int 1989; 35: 1336.

16. Cohen EP, Lawton CA, Moulder JE, et al. Clinical course of late-onset bone marrow transplant nephropathy. Nephron 1993; 64: 626.

17. van der Plas RM, Schiphorst ME, Huizinga EG, et al. von Willebrand factor proteolysis is deficient in classic, but not in bone marrow transplantation-associated, thrombotic thrombocytopenic purpura. Blood 1999; 93: 3798

18. Elliott MA, Nichols WL, Plumhoff EA, et al. Posttransplantation thrombotic thrombocytopenic purpura: A single-center experience and a contemporary review. Mayo Cl Proc 2003; 78: 421.

19. Peyvandi F, Siboni S, Lambertenghi-Delliers G, et al. Prospective study on the behavior of the metalloprotease ADAMTS13 and of von Willebrand factor after bone marrow transplantation. Br J Haematol 2006; 134: 187 .

\section{Transplantation Mission Statement}

The most cited and influential journal in the field (with over 20,000 citations a year),

Transplantation is published twice monthly and provides extensive coverage of the most important advances in transplantation.

Using an electronic on-line submission and peer review tracking system, Transplantation is committed to rapid review and publication. The average time to first decision is now less than 21 days. Time to publication of accepted manuscripts continues to be shortened, with the Editorial team committed to a goal of 3 months from acceptance to publication. Consistently ranked among the top journals in Transplantation, Surgery and Immunology (Journal Citation Reports), the journal covers areas including:

- CELL THERAPY AND ISLET TRANSPLANTATION

- CLINICAL TRANSPLANTATION

- EXPERIMENTAL TRANSPLANTATION

- IMMUNOBIOLOGY AND GENOMICS

- XENOTRANSPLANTATION 